\title{
The effects of restricted feeding on the ultradian rhythm of corticosterone secretion and steroidogenesis in the rat adrenal
}

\author{
V. Pyc, F. Spiga and S. Lightman \\ School of Clinical Sciences, University of Bristol, Dorothy Hodgkin Building, BS1 3NY, UK
}

Glucocorticoids are vital hormones that are released in response to stress to regulate a number of physiological functions, including metabolism, inflammation and cardiovascular activity. Their release is characterized by an ultradian (pulsatile) pattern of hormone secretion which disturbance has been linked to obesity ${ }^{(1)}$. The literature provides evidences suggesting that fasting can affect glucocorticoid secretion both by increasing hormone synthesis in the adrenal and by increasing corticosterone half-life ${ }^{(2)}$. This project was aimed to further elucidate the mechanisms underlying the observed changes in corticosterone secretion.

An automated blood sampling system was used to collect blood samples over a period of $24 \mathrm{hrs}$ from rats that were allocated to one of the three experimental groups: ad libitum, fasted and fed group. The blood plasma was further analysed by a radioimmune corticosterone assay to quantify the corticosterone levels. By using a reverse transcriptase quantative polymerase chain reaction the gene expression in the adrenal gland was measured from rats subjected to the three experimental conditions.

This study has established that, compared to ad libitum fed animals, fasting increases, while feeding decreases, basal corticosterone levels (Figure 1A), but neither conditions affect the hormone response to a mild stressor. Moreover, the increased corticosterone secretion in fasted rats was paralleled by a decrease of the inhibitory protein DAX-1 (Figure 1B), that is known to be crucial for the expression of key steroidogenic genes in the adrenal. A change of only one steroidogenic factor suggests a more complex adrenal response to fasting, possibly involving other metabolites acting on the gland.
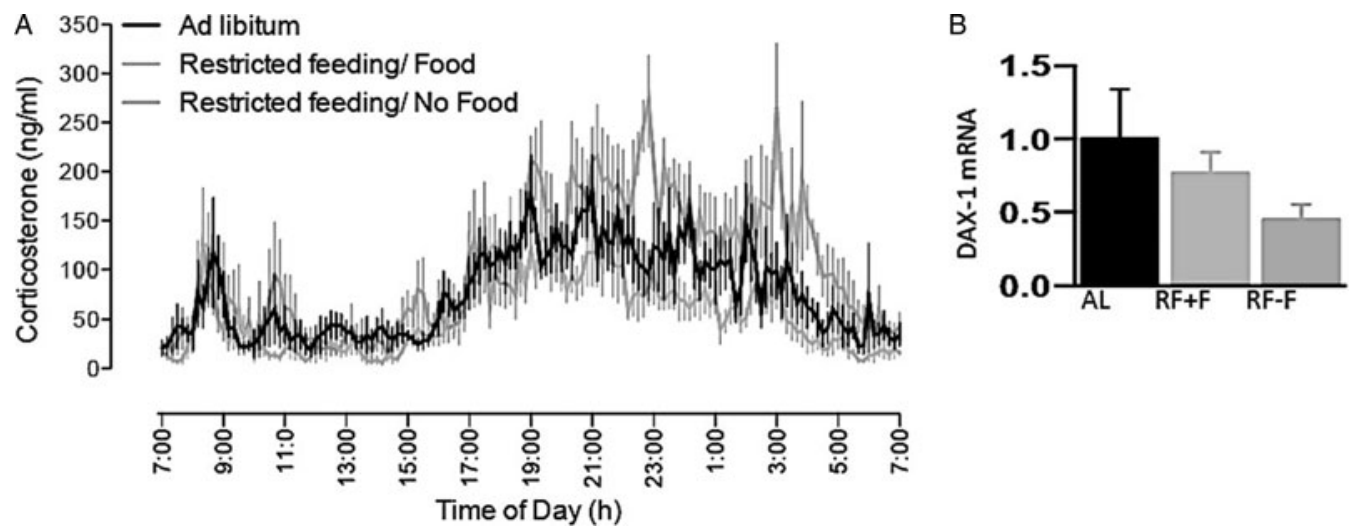

Fig. 1A. The effects of fasting and feeding on the rhythm of corticosterone secretion over $24 \mathrm{hrs}$. Food was administered Ad libitum, or on a restricted regimen (RF) 12 h/day (from 19:00; RF+Food) or rats were fasted for $24 \mathrm{~h}$ (RF-Food). Fig. 1B. DAX-1 mRNA levels in Ad libitum fed rats and in rats kept on the restricted feeding regimen with or without food; $\mathrm{P}=0.02 \mathrm{RF}+$ Food versus RF-Food.

1. Spiga F \& Lightman SL (2015) Mol Cell Endocrinol 408, 227-34.

2. Chang LL et al. (2002) J Investig Med 50, 86-94. 